\title{
Distinct Influence of Hand Posture on Cortical Activity during Human Grasping
}

\author{
Monica A. Perez ${ }^{1}$ and John C. Rothwell ${ }^{2}$ \\ ${ }^{1}$ University of Pittsburgh, Department of Physical Medicine and Rehabilitation, Center for the Neural Basis of Cognition, Systems Neuroscience Institute, \\ Pittsburgh, Pennsylvania 15261, and 2Institute of Neurology, University College London, London WC1N 3BG, United Kingdom
}

We recently showed that subcortical circuits contribute to control the gain of motor cortical inputs to spinal motoneurons during precision grip of a small object. Here, we examine whether the involvement of the motor cortex could be revealed by grasping with different hand postures. Using noninvasive cortical, cervicomedullary, and peripheral nerve stimulation we examined in humans motorevoked potentials (MEPs) and the activity in intracortical circuits (suppression of voluntary electromyography) and spinal motoneurons (F-waves) in intrinsic hand muscles when grasping a $6 \mathrm{~mm}$ cylinder with the index finger and thumb while the hand was held in the neutral position or during full pronation and supination. We demonstrate that the size of cortically evoked MEPs in the first dorsal interosseous, but not in the abductor pollicis brevis and abductor digit minimi muscles, was reduced to a similar extent during grasping with the hand pronated or supinated compared with the neutral position. Notably, the suppression of MEPs was present from the MEP onset, suggesting that indirect corticospinal pathways were less likely to be involved than direct connections. There was less intracortical inhibition targeting the first dorsal interosseous during hand pronation and supination compared with neutral and this negatively correlated with changes in MEP size. In contrast, cervicomedullary MEPs and F-waves remained unchanged across conditions, as did MEPs evoked during unopposed weak flexion of the index finger. Our findings reveal a distinct influence of the posture of the hand on the activity of cortical pathways controlling different hand muscles during grasping.

Key words: corticomotoneuronal cells; corticospinal tract; intracortical inhibition; precision grip; primary motor cortex; voluntary drive

\section{Introduction}

Anatomical and electrophysiological studies have shown that corticospinal neurons from the motor cortex, particularly corticomotoneuronal cells, make a crucial contribution to the execution of grasping with the index finger and thumb (Lemon, 2008). Recent studies showed that subcortical networks also modulate the excitability of spinal motoneurons during a precision grip task in nonhuman primates (Takei and Seki, 2010, 2013) and in humans (Bunday et al., 2014). However, the role of the cortical and subcortical networks may differ. The activity of neurons from the motor cortex reflects, in part, the generation of actions in terms of extrinsic space coordinates related to hand motion (Georgopoulos et al., 1982; Kakei et al., 1999), whereas spinal interneurons are more widely activated during voluntary movement (Fetz et al., 2000). The aim of the present experiments was to provide further evidence for a dual level of cortical and spinal control in human precision grip.

\footnotetext{
Received 0ct. 8, 2014; revised Feb. 12, 2015; accepted Feb. 13, 2015.

Author contributions: M.A.P. and J.C.R. designed research; M.A.P. and J.C.R. performed research; M.A.P. and J.C.R. contributed unpublished reagents/analytic tools; M.A.P. and J.C.R. analyzed data; M.A.P. and J.C.R. wrote the paper.

The authors declare no competing financial interests.

Correspondence should be addressed to Dr Monica A. Perez, Department of Physical Medicine and Rehabilitation, Center for the Neural Basis of Cognition, Systems Neuroscience Institute, University of Pittsburgh, Pittsburgh, Pennsylvania 15261. E-mail: perezmo@pitt.edu.

DOI:10.1523/JNEUROSCI.4170-14.2015

Copyright $\odot 2015$ the authors $\quad 0270-6474 / 15 / 354882-08 \$ 15.00 / 0$
}

In a previous study (Bunday et al., 2014), we explored the role of spinal, probably GABAb-ergic, circuits in modulating the gain of cortical input to spinal motoneurons during static precision grip of a small object. The later portion of the electromyographic (EMG) activity evoked during precision grip by transcranial magnetic stimulation (TMS) of motor cortex or by transmastoidal electrical stimulation of corticospinal axons in the brainstem was smaller compared with that evoked during unopposed index finger abduction. We proposed that this type of precision grip suppressed transmission in probable disynaptic or polysynaptic corticospinal inputs to hand motoneurons, effectively increasing the role of direct monosynaptic corticomotoneuronal inputs. However, there was no evidence for changes in excitability within the motor cortex itself. The present experiments show that such effects can be revealed by performing the same grip in different hand postures.

In nonhuman primates, evidence showed that the discharge rate of corticospinal cells varies by changing the direction of a finger movement (Schwartz, 1992) and the position of the wrist joint (Thach, 1978). The coordinated activity between the wrist and fingers during precision grip relies on the integrity of the motor cortex (Rouiller et al., 1998). In agreement, human studies showed that wrist flexion and extension result in distinct changes in corticospinal excitability in the index finger muscle during a precision grip task (Gagné and Schneider, 2007). Indeed, corticospinal excitability in hand muscles is also modulated to a different extent by changing the orientation of the shoulder joint in 
opposite directions (Dominici et al., 2005). However, these latter studies in humans did not distinguish between changes in excitability at cortical or spinal levels, referring only to changes in "corticospinal excitability." In the present experiments, we therefore probed cortical and spinal changes in motor transmission when participants grasped a small object using a precision grip with the hand oriented in the vertical compared with the horizontal plane. Because intracortical circuits contribute to modulate corticospinal drive during hand motor skills (Nordstrom and Butler, 2002) and the directional tuning of corticospinal cells (Mahan and Georgopoulos, 2013), we also expected accompanying changes in the activity of intracortical circuits, as measured by the suppression of voluntary electromyography (svEMG; Butler et al., 2007).

\section{Materials and Methods}

Subjects. Nineteen healthy control subjects (mean age $=29.5 \pm 8.4$ years; 11 male, 3 left-handed) participated in the study. All subjects gave informed consent to experimental procedures, which were approved by the local ethics committee at the University College London and at the University of Pittsburgh. All subjects participated in two experimental session and a subgroup of them in three sessions.

Recordings. In all subjects EMG was recorded from the first dorsal interosseous (FDI), abductor pollicis brevis (APB), and the abductor digiti minimi (ADM) of the dominant side through surface electrodes secured to the skin over the belly of each muscle $(\mathrm{Ag}-\mathrm{AgCl}, 10 \mathrm{~mm}$ diameter). In an additional control experiment $(n=7)$, EMG was also recorded from the pronator teres (PT), long head of the biceps (LB), and brachioradialis (BR) of the dominant side. The signals were amplified, filtered $(30-1000 \mathrm{~Hz}$ ), and sampled at $5 \mathrm{kHz}$ for off-line analysis (Cambridge Electronic Design micro1401 with Signal software v4.1).

Experimental setup. Fifteen subjects performed grasping (Fig. 1) while seated in an armchair with both arms flexed at the elbow by $90^{\circ}$ with forearms resting on a pillow. During grasping, subjects were instructed to hold a small cylinder (diameter: $6 \mathrm{~mm}$; length: $31 \mathrm{~mm}$; mass: $1.36 \mathrm{~g}$ ) between the index finger and thumb while the hand was held in the neutral position (Fig. 1A) or during full pronation (Fig. 1B) and supination (Fig. 1C). The instruction for the subjects was to maintain the cylinder in each position with the minimal amount of EMG activity in both fingers referred to as "natural precision grip". At the start of the experiment subjects performed two brief maximal voluntary contractions (MVCs; 3-5 s) with the index finger into abduction, thumb into abduction, and with the little finger into adduction, separated by $30-60 \mathrm{~s}$ of rest. MVCs were quantified using background EMG activity in each muscle tested. During index and little finger abduction, subjects were instructed to press with the index and little finger against a custom lever or the experimenter into the abduction direction with the forearm pronated and supinated, respectively, and the hand stabilized by the experimenter or the wrist restrained by straps. During thumb abduction, subjects were instructed to press with the thumb against the experimenter into the abduction direction with the forearm supinated and the hand stabilized by the experimenter or the wrist restrained by straps. A familiarization trial was completed at the beginning of each experiment to ensure that subjects were able to match EMG activity in the FDI muscle during all tasks. For this purpose, mean rectified EMG activity in the FDI muscle was shown online on a computer screen located in front of subjects using Signal software. Here, individual frames of $4 \mathrm{~s}$ duration showed the mean rectified EMG activity in the FDI muscle for $1 \mathrm{~s}$ trial by trial and a cursor region option showed the numeric value for EMG activity in individual trials. During familiarization, subjects were asked to hold the pin between the index and thumb in each of the positions and then the minimal amount of EMG activity at which individuals could maintain comfortable similar background in the FDI muscle across gripping tasks was determined. Tasks were completed in a pseudorandomized order. Mean rectified EMG activity from the FDI muscle was displayed continuously on a computer screen positioned in front of the subjects and verbal feedback was provided to ensure that physiological measurements in the
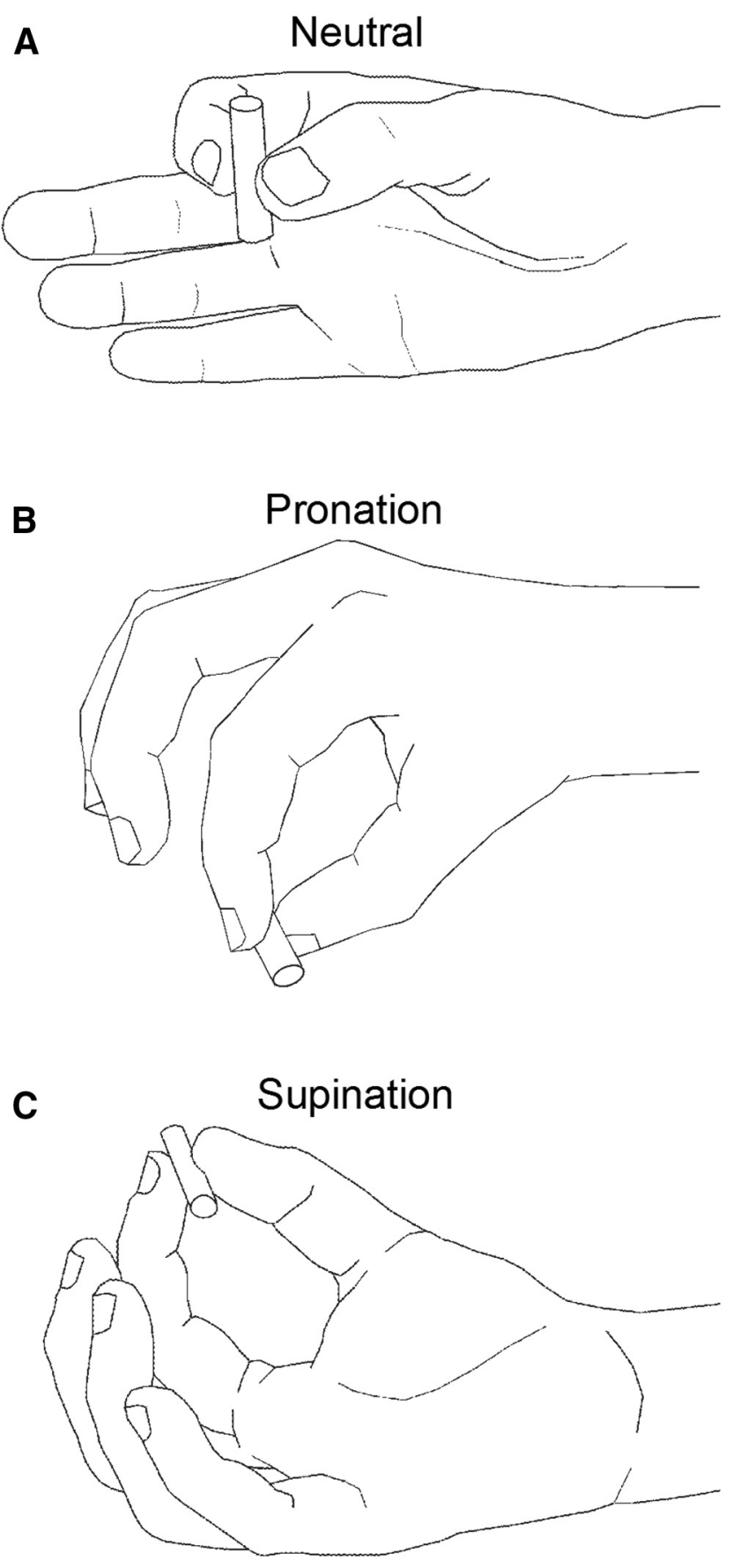

Figure 1. Experimental setup. Schematic of the hand posture when grasping a small cylinder between the index finger and thumb with the hand in the neutral position $(\boldsymbol{A})$ or during full pronation $(\boldsymbol{B})$ and supination $(\boldsymbol{C})$.

FDI were acquired at similar EMG background at all times. In a control experiment $(n=8)$, the elbow was flexed and the hand was maintained in the vertical position with the hand straight. Here, a small amount of force was generated with the index finger into flexion with the forearm in neutral position, pronated, and supinated to avoid the effect of gravity and test for nonspecific effects related to hand posture and force direction. A total of $8.1 \%$ trials in which mean rectified EMG activity exceeded $\pm 1.5 \mathrm{SD}$ of the average contracting mean rectified EMG, measured 100 $\mathrm{ms}$ before the stimulus artifact, were excluded from further analysis. The coefficient-of-variation (CV) of EMG (SD/mean EMG) signals was measured from the FDI, APB, and ADM muscles during all tasks. EMG was rectified and averaged $100 \mathrm{~ms}$ before TMS for each trial. 
TMS. Transcranial magnetic stimuli were delivered from a Magstim 200 stimulator (Magstim) through a figure-eight coil with a monophasic current waveform. TMS was delivered to the optimal scalp position for activating the FDI ensuring the motor-evoked potentials (MEPs) were present in APB and ADM muscles. To identify the optimal scalp position for the FDI the coil was held tangential to the scalp with the handle pointing backward and $45^{\circ}$ away from the midline. With this coil position the current flowed in a posterior-anterior direction and probably produced D and early I wave activation of corticospinal neurons (Sakai et al., 1997). The TMS coil was held to the head of the subject by one of the experimenters. TMS measurements included motor evoked potentials elicited by motor cortical (MEPs) and cervicomedullary (CMEPs) stimulation, resting motor threshold (RMT), and suppression of voluntary EMG by subthreshold TMS (svEMG).

$M E P s$. RMT was defined as the minimal stimulus intensity required to induce MEPs $\geq 50 \mu \mathrm{V}$ peak-to-peak amplitude above the resting background EMG in at least three of five consecutive trials in resting muscle (Rothwell et al., 1999). During grasping, MEPs were tested using RMT intensity. Single TMS pulses were delivered at $4 \mathrm{~s}$ intervals in sets of 10 separated by resting periods as needed. Thirty MEPs were tested during grasping in each position in a pseudorandomized order.

CMEPs. The corticospinal tract was stimulated at the cervicomedullary level $(n=9)$ by using a double-cone magnetic coil over the back of the head. The stimulation intensity was set to elicit a CMEP in the FDI muscle between 0.5 and $1 \mathrm{mV}(86.6 \pm 14.1 \%$ of maximal stimulator output) during grasping in the neutral position. Thirty CMEPs were tested during grasping in each position in a pseudorandomized order.

F-waves. Motoneuron excitability (reflected by F-wave amplitude and persistence) was measured ( $n=7)$ by using supramaximum stimulus intensity to the ulnar nerve on the ulnar side of the wrist (200 $\mu$ s pulse duration; DS7A, Digitimer) in all muscles tested. The anode and cathode were $3 \mathrm{~cm}$ apart and $1 \mathrm{~cm}$ in diameter with the cathode positioned proximally. The stimuli were delivered at $1 \mathrm{~Hz}$ at an intensity of $150 \%$ of the M-max. For each trial, we quantified peak-to-peak amplitude (expressed relative to the $\mathrm{M}$-max) and $\mathrm{F}$-wave persistence (number of F-waves present in each set). Mean background EMG activity 100 ms before stimulus artifact was subtracted from each F-wave. We identified an F-wave as present if a response with a proper latency (minimum of $20 \mathrm{~ms}$ for FDI, APB, and ADM) had an amplitude $\geq 20 \mu \mathrm{V}$ above background. Sixty F-waves were tested in each condition. Measurements were tested during grasping in each position in a pseudorandomized order. F-waves were also tested in the APB muscles by moving the stimulating electrode to the median nerve at the medial part of the wrist using supramaximum stimulus intensity in each position. We observed the same result in the F-waves in the APB muscle with ulnar and median nerve stimulation.

svEMG. TMS at an intensity below MEP threshold applied during voluntary contraction suppresses EMG activity (svEMG). It is thought that the svEMG activity reflects activation of cortical inhibitory mechanisms (Butler et al., 2007). The svEMG $(n=10)$ was first measured in the FDI muscle during grasping with the hand in the neutral position. At first, the stimulus intensity was increased in small steps until the svEMG was present without evoking short-latency facilitation. Using the same stimulus intensity the svEMG was also present on the APB $(n=8)$ and $\operatorname{ADM}(n=4)$. The onset of the svEMG was determined by visual inspection and by using a horizontal cursor showing the mean rectified EMG before the TMS artifact as a reference. svEMG onset was defined as the time-point when the mean rectified EMG activity dropped below the mean (for $10 \mathrm{~ms}$ ) and the end of the svEMG as the time-point when the EMG returned through this level. The area of the svEMG was measured between the onset and offset of the svEMG using the following formula: svEMG area $=[$ (background mean rectified $\mathrm{EMG} \times \mathrm{svEMG}$ duration) - au_svEMG], where background EMG is the mean amplitude rectified EMG for 100 ms prestimulus period, and au_svEMG is the area under the rectified svEMG. After that, the svEMG area was normalized against the prestimulus EMG level [svEMG area normalized to prestimulus $\mathrm{EMG}=$ svEMG area/(background mean rectified $\mathrm{EMG} \times$ mean duration of svEMG); Bunday et al., 2014]. Mean duration (FDI = $20.6 \pm 6.5 \mathrm{~ms}, \mathrm{APB}=19.7 \pm 7.1 \mathrm{~ms}, \mathrm{ADM}=12.6 \pm 3.1 \mathrm{~ms} ; p=0.15)$ and latency $(\mathrm{FDI}=32.0 \pm 9.4 \mathrm{~ms}, \mathrm{APB}=31.2 \pm 6.9 \mathrm{~ms}, \mathrm{ADM}=32.3 \pm$
Table 1. EMG activity and CV

\begin{tabular}{llll}
\hline & Neutral & Pronation & Supination \\
\hline Mean rectified EMG $(\mathrm{mV})$ & & & \\
FDI & $0.022 \pm 0.02$ & $0.022 \pm 0.01$ & $0.021 \pm 0.02$ \\
APB & $0.022 \pm 0.032$ & $0.008 \pm 0.008$ & $0.030 \pm 0.01$ \\
ADM & $0.009 \pm 0.005$ & $0.012 \pm 0.01$ & $0.009 \pm 0.005$ \\
PT & $0.009 \pm 0.01$ & $0.009 \pm 0.006$ & $0.008 \pm 0.009$ \\
LB & $0.005 \pm 0.003$ & $0.005 \pm 0.004$ & $0.007 \pm 0.004$ \\
BR & $0.007 \pm 0.004$ & $0.007 \pm 0.003$ & $0.006 \pm 0.001$ \\
CV EMG & & & \\
FDI & $1.1 \pm 0.2$ & $1.1 \pm 0.3$ & $1.1 \pm 0.3$ \\
APB & $1.1 \pm 0.1$ & $1.0 \pm 0.1$ & $1.1 \pm 0.2$ \\
ADM & $1.4 \pm 0.2$ & $1.3 \pm 0.2$ & $1.3 \pm 0.2$ \\
PT & $0.5 \pm 0.2$ & $0.4 \pm 0.1$ & $0.4 \pm 0.2$ \\
LB & $0.4 \pm 0.1$ & $0.3 \pm 0.1$ & $0.4 \pm 0.2$ \\
BR & $0.3 \pm 0.03$ & $0.3 \pm 0.1$ & $0.3 \pm 0.05$ \\
\hline
\end{tabular}

$7.3 \mathrm{~ms} ; p=0.8$ ) of svEMG across muscles were similar in all tasks. The interstimulus interval for TMS pulses was $1 \mathrm{~s}$. Seventy-five trials were tested in each condition.

Data analysis. Two-way repeated-measures ANOVAs were performed to determine the effect of task (neutral, pronation, supination) and muscle (FDI, APB, ADM) on cortical MEPs, CMEPs, F-wave persistence and amplitude, background mean EMG activity, and CV. The same analysis was conducted to assess the effects of task and muscle controls (PT, LB, BR) background mean EMG activity. Two-way ANOVAs were performed to test the effect of task and muscle on svEMG. Additional repeated ANOVAs were performed on each group separately as needed. Pairwise comparisons were used to test for significant comparisons between intensities. A Pearson correlation analysis was used as needed. Significance was set at $p<0.05$. Group data are presented as the mean \pm $\mathrm{SD}$ in the text.

\section{Results \\ EMG}

Repeated-measures ANOVA showed a significant effect of muscle $\left(F_{(2,56)}=17.4, p<0.001\right)$, but not task $\left(F_{(2,56)}=1.7, p=0.22\right)$ nor in their interaction $\left(F_{(4,56)}=1.0, p=0.32\right)$ on mean rectified EMG activity. Subjects were able to maintain similar EMG activity in the FDI $(p=0.31), \operatorname{APB}(p=0.28)$, and $\operatorname{ADM}(p=0.37)$ across tasks. We found no effect of muscle $\left(F_{(2,24)}=1.8, p=0.2\right)$, task $\left(F_{(2,24)}=0.6, p=0.5\right)$ nor in their interaction $\left(F_{(4,24)}=2.2\right.$, $p=0.1$ ) on mean rectified EMG activity (Table 1$)$. The CV was larger for the ADM compared with the FDI $(p=0.03)$ and APB $(p=0.02)$ muscles (Table 1$)$ but no differences were found across tasks $\left(F_{(2,56)}=1.7, p=0.22\right)$.

\section{MEPs}

Figure 2 illustrates MEPs from a representative participant tested during grasping with the hand in different directions. Note that the size of MEPs in the FDI but not in the APB and ADM muscles decreased when the hand was pronated and supinated compared with the neutral position. Repeated-measures ANOVA showed a significant effect of task $\left(F_{(2,56)}=17.4, p<0.001\right)$, muscle $\left(F_{(2,56)}=\right.$ $87.9, p<0.001)$ and in their interaction $\left(F_{(4,56)}=16.7, p<0.001\right)$ on MEPs size. The FDI MEP size was significantly decreased when the hand was pronated $(76.2 \pm 17.6 \%)$ and supinated $(71.5 \pm 20.4 \%)$ compared with the neutral position $(p<0.001)$. No differences were found in the APB and ADM MEP size across tasks. A control experiment, where individuals needed to exert EMG activity into index finger flexion (neutral $=0.024 \pm 0.01$ $\mathrm{mV}$, pronation $=0.025 \pm 0.01 \mathrm{mV}$, supination $=0.020 \pm 0.01$ $\mathrm{mV}, p=0.2$ ), whereas the wrist was held in different orientations with the hand elevated, revealed no differences in MEP size across 

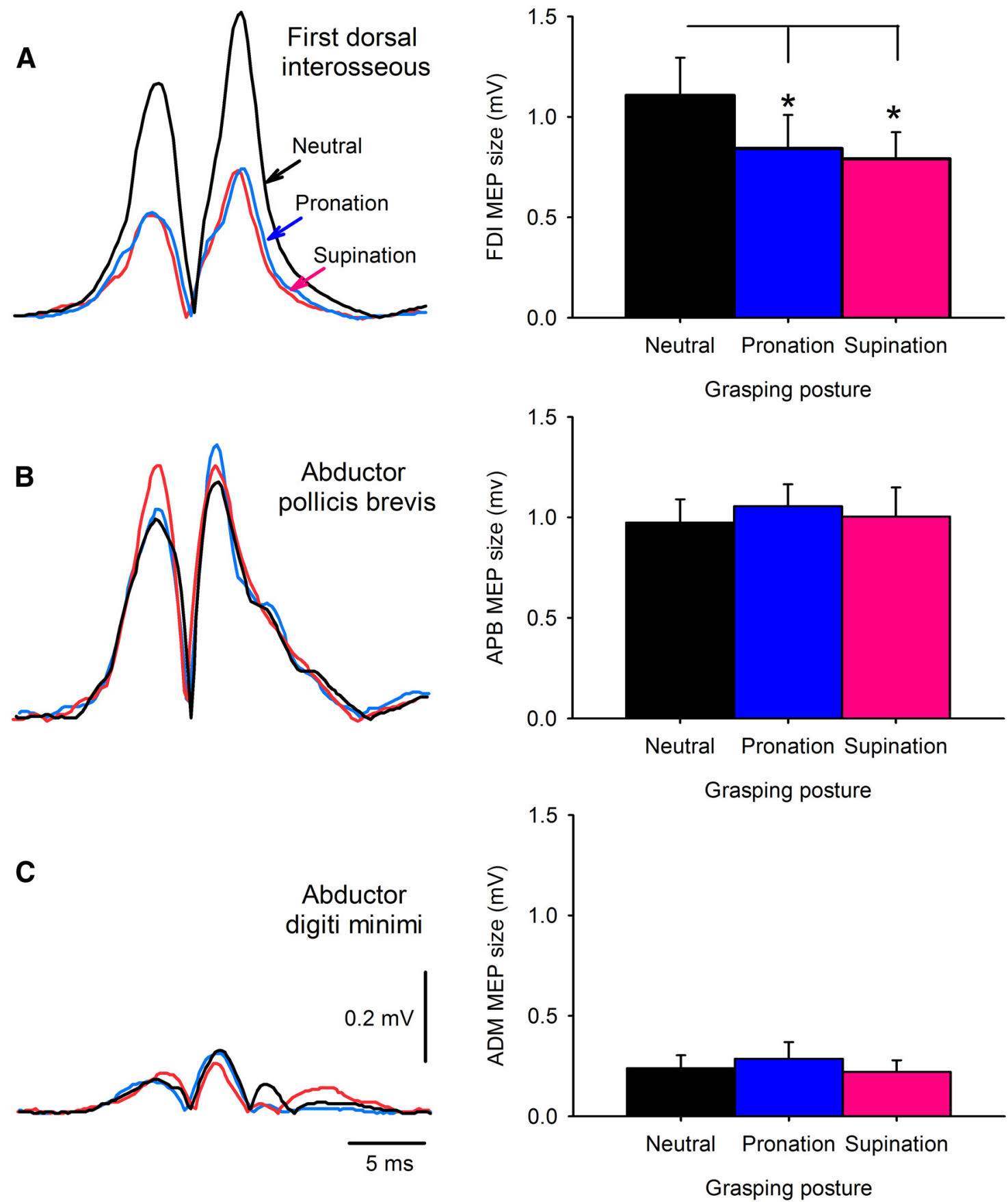

Figure 2. MEPs tested in the first dorsal interosseous $(\boldsymbol{A})$, abductor pollicis brevis $(\boldsymbol{B})$, and abductor digiti minimi $(\boldsymbol{C})$ muscles in a representative subject during grasping with the hand in the neutral position (black traces), pronation (blue traces), and supination (red traces). Traces show the average 30 rectified MEPs. The arrow indicated the onset of the MEP suppression. Group data are shown in bar graphs $(n=15)$. The abscissa shows the hand posture tested (neutral, pronation, supination). The ordinate shows the MEP size in millivolts in each position tested. Error bars indicate $\mathrm{SE} ;{ }^{*} p<0.05$.

muscles $\left(F_{(4,28)}=1.1, p=0.4\right)$. The size of the FDI MEPs was $0.92 \pm 0.6 \mathrm{mV}$ when force was directed in the neutral position, $0.89 \pm 0.5 \mathrm{mV}$ in the pronation and $0.96 \pm 0.6 \mathrm{mV}$ in the supination direction.

\section{SvEMG}

Figure $3 A$ illustrates mean rectified EMG in the FDI muscle during subthreshold TMS in a representative subject. Note that at similar levels of mean rectified EMG activity before TMS artifact, there is less EMG suppression (Fig. 3A, period between dotted lines) when grasping with the hand pronated or supinated com- pared with the neutral position. ANOVA tests showed a significant effect of task $\left(F_{(2,57)}=3.2, p=0.04\right)$, muscle $\left(F_{(2,57)}=13.8\right.$, $p<0.001)$, and in their interaction $\left(F_{(4,57)}=3.7, p<0.01\right)$ on the svEMG area. In the FDI muscle, the svEMG area was less suppressed during pronation ( $81.2 \pm 10.8 \%$ of baseline EMG levels) and supination $(83.8 \pm 7.2 \%)$ compared with the neutral position $(75.4 \pm 8.1 \%$; Fig. $3 B)$. The same result was observed if the same window was used for analysis across conditions $\left(F_{(2,18)}=\right.$ $10.6, p<0.01$; svEMG area during pronation $=79.4 \pm 10.8 \%$ of baseline EMG; supination $=83.1 \pm 7.3 \%$; neutral position $=$ $74.3 \pm 8.1 \%)$. In contrast, no differences were observed in the 

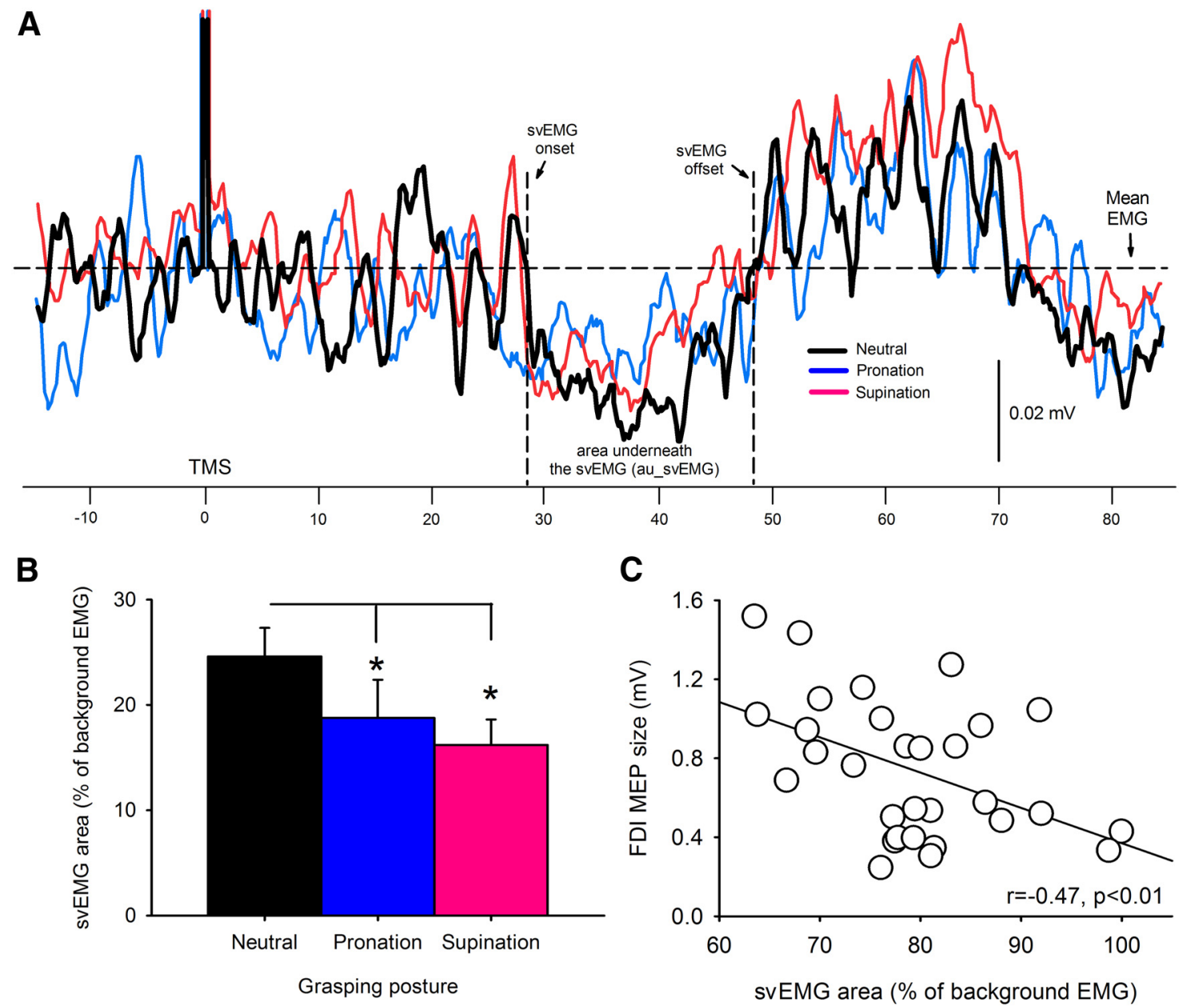

Figure 3. SvEMG. A, Raw EMG data in a representative subject showing the svEMG elicited by subthreshold TMS recorded in the first dorsal interosseous muscle during grasping (neutral position, black traces; pronation, blue traces; supination, red traces). Seventy-five traces were averaged in each condition. The onset and offset of the svEMG is shown between dotted lines and the area underneath the au_svEMG (see Materials and Methods for detailed description). The horizontal dotted line showed the mean rectified EMG activity $100 \mathrm{~ms}$ before TMS artifact. $\boldsymbol{B}$, Graph shows group data $(n=10)$. The abscissa shows the grasping posture tested (neutral, pronation, supination) and the ordinate shows the normalized area of the svEMG (see Materials and Methods). $\boldsymbol{C}$, Graph shows a correlation analysis between MEPs size and the svEMG with responses normalized to the mean value across conditions for each measurement. Error bars indicate $S E ;{ }^{*} p<0.05$.

svEMG area in the APB (neutral $=78.8 \pm 8.4 \%$ of baseline EMG; pronation $=77.1 \pm 8.7 \%$ of baseline EMG; supination $=80.2 \pm$ $6.5 \%$ of baseline EMG; $p=0.4$ ) and $\mathrm{ADM}$ (neutral $=83.8 \pm$ $8.4 \%$ of baseline EMG; pronation $=84.5 \pm 9.8 \%$ of baseline EMG; supination $=82.5 \pm 5.9 \%$ of baseline EMG; $p=0.5$ ) muscles across conditions. The onset $\left(F_{(4,57)}=1.6, p=0.2\right)$ and duration $\left(F_{(4,57)}=1.0, p=0.4\right)$ of the svEMG and background rectified EMG area $\left(F_{(4,57)}=1.9, p=0.1\right)$ did not differ between conditions across muscles. A negative correlation was found between FDI svEMG area and MEPs size during grasping in all conditions tested ( $r=-0.47, p<0.01$; Fig. $3 C$ ). Thus, individuals with smaller MEPs were those who showed less suppression during the svEMG.

\section{CMEPs}

Figure 4 illustrates an example of CMEPs in the FDI muscle in a representative participant. Note that CMEP size remains similar across tasks. In all subjects tested, the latency of CMEPs was significantly shorter than MEPs elicited by TMS (CMEPs $=17.7 \pm 1.3 \mathrm{~ms}$, MEPs $=21.2 \pm 1.0 \mathrm{~ms} ; p<0.01)$ indicating that the stimulation activated corticospinal axons distal to the motor cortex. ANOVA tests showed no effect of task $\left(F_{(2,60)}=0.2, p=0.7\right)$, muscle $\left(F_{(2,60)}\right.$ $=0.3, p=0.7)$ and in their interaction $\left(F_{(4,60)}=1.2, p=0.3\right)$ on
CMEP size indicating that CMEPs remained unchanged during grasping in all conditions tested. Background EMG amplitude was similar between conditions across muscles $\left(F_{(4,60)}=1.2, p=0.3\right)$.

\section{F-waves}

The maximum $\mathrm{M}$-wave was similar across muscles (FDI = $20.2 \pm 2.5 \mathrm{mV}, \mathrm{APB}=17.2 \pm 2.2 \mathrm{mV}, \mathrm{ADM}=18.2 \pm 1.6 \mathrm{mV}$; $p=0.1)$. Repeated-measures ANOVA showed no effect of task $\left(F_{(2,24)}=1.6, p=0.2\right)$, muscle $\left(F_{(2,24)}=0.7, p=0.5\right)$, and in their interaction $\left(F_{(4,24)}=0.3, p=0.8\right)$ on F-wave amplitude. Similarly, when comparing F-wave persistence we found no effect of task $\left(F_{(2,24)}=1.1, p=0.4\right)$, muscle $\left(F_{(2,24)}=0.6, p=0.5\right)$, nor in their interaction $\left(F_{(4,24)}=0.9, p=0.5\right)$. Background EMG amplitude was similar across tasks in all muscles tested $\left(F_{(2,24)}=1.0\right.$, $p=0.4)$.

\section{Discussion}

Our findings demonstrate that excitability of corticospinal and cortical circuits involved in controlling the FDI muscle during a precision grip is influenced by the posture of the hand. We found that the size of MEPs evoked by cortical stimulation in the FDI, but not in the APB and ADM muscles, was reduced during grasping when the hand was pronated and supinated compared with 
A
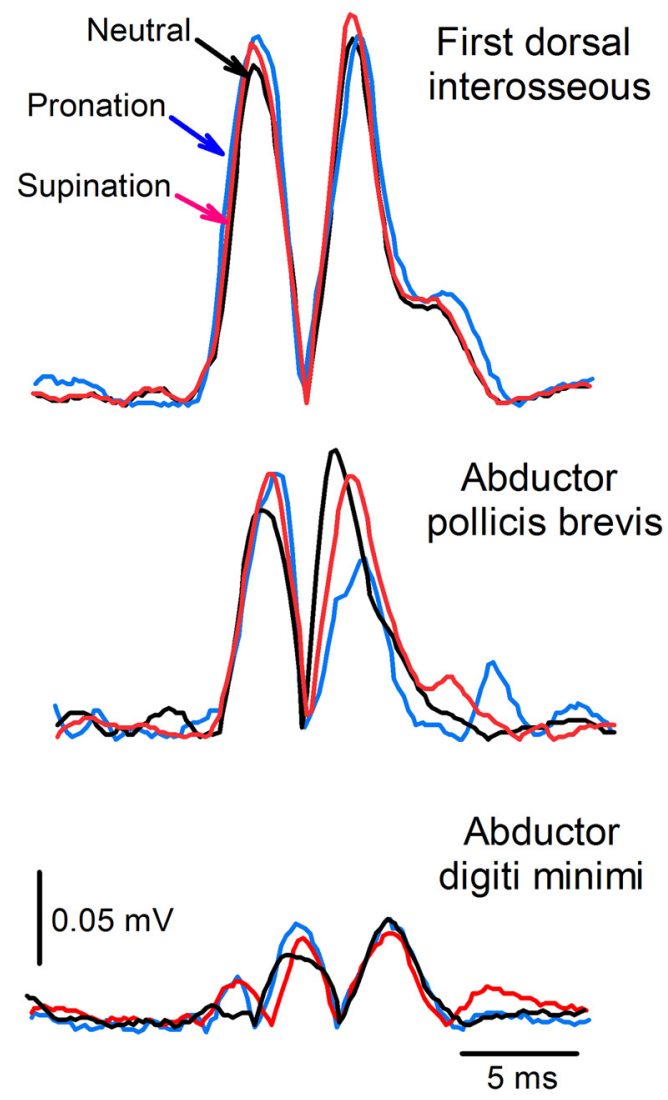

B

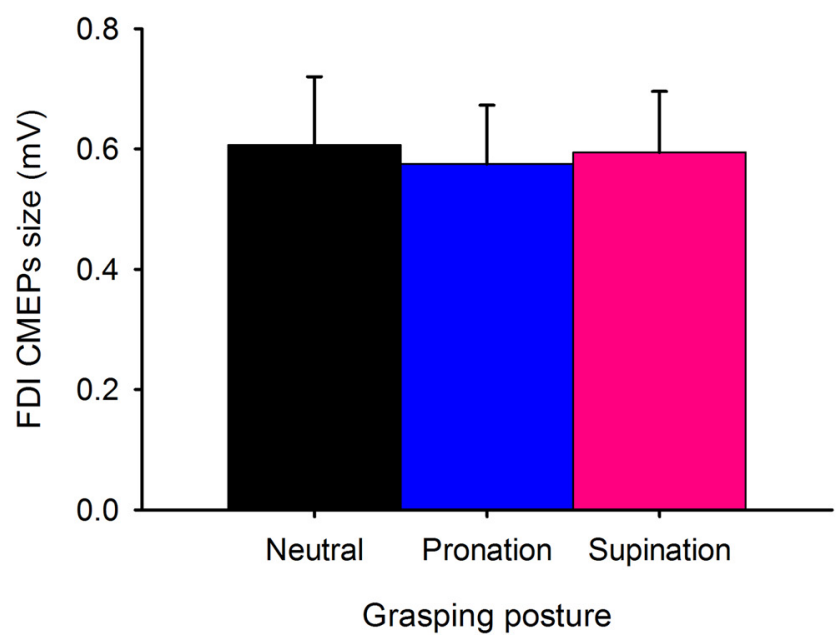

Figure 4. CMEPs. A, Raw EMG data in a representative subject showing MEPs elicited by cervicomedullary stimulation recorded from the first dorsal interosseous, abductor pollicis brevis, and abductor digiti minimi muscles during grasping. Traces show the average 30 rectified CMEPs. $\boldsymbol{B}$, In the graph $(n=9)$, the abscissa shows the grasping posture tested (neutral, pronation, supination) and the ordinate shows the MEP size in millivolts in each position tested. Error bars indicate $\mathrm{SE}_{;}{ }^{*} p<0.05$.

the neutral position. Notably, MEP suppression was present from the MEP onset, suggesting that indirect corticospinal pathways were less likely to be involved than direct connections. Intracortical inhibition decreased to a similar extent during hand pronation and supination and was inversely correlated with changes in MEP size. In contrast, cervicomedullary MEPs and F-waves remained unchanged across conditions, as did MEPs evoked during unopposed finger flexion. Thus, the effect of hand posture on the excitability of corticospinal output to hand muscles varies according to the task being performed, as well as the role of the muscle in the task.

\section{Orientation of the hand during grasping}

A first intriguing question that arises from our results is why MEPs were suppressed in the FDI but not in the APB muscle during object grasping. Previous evidence showed that the majority of intrinsic hand muscles display activity that correlates with low levels of precision grip force (Maier and HeppReymond, 1995). Therefore, if our effect was only related to changes in the grasping direction one would expect that MEPs, at least in the APB, would also change. The lack of change in MEP size in the APB muscle indicates that other factors might have also influenced our results. Biomechanically, during grasping with the index finger and thumb, the FDI contributes to flexion at the metacarpal joint, which is one of the primary actions for this muscle; whereas the APB and other thumb muscles have a variety of actions. Indeed, it has been shown that during a precision grip the intrinsic muscles of the thumb behave quite differently from each other (Maier and Hepp-Reymond, 1995). The function of the APB in grasping is less clear because its pulling directions do not allow a direct grip force production, suggesting that this muscle might have a more stabilizing role (Chao et al., 1976), whereas the FDI might contribute to the fine grading of forces during grasping (Maier and Hepp-Reymond, 1995).

Our results suggest that muscles with different contributions to a task are affected in different ways by hand posture. Physiological differences existing in connections from the motor cortex to the FDI and APB could also account for this. In nonhuman primates, a single corticomotoneuronal cell can facilitate intrinsic hand muscles with close functional relation but spike-evoked responses from the same corticomotoneuronal cell were not found in the FDI and APB muscles (Buys et al., 1986). Thus, if corticomotoneuronal projections contributed to our effects, as suggested by the fact that FDI MEP suppression was present from MEP onset, it would be quite possible for MEPs to be modulated differentially in the two muscles. In our previous study where we showed the contribution of subcortical pathways to precision grip, the suppression of the MEP was evident $1-3 \mathrm{~ms}$ after its onset (Bunday et al., 2014) supporting the notion that MEPs can be used to detect the effects of inputs from more and less direct pathways to spinal motoneurons during our task.

A second intriguing question is why MEPs in the FDI muscle were suppressed when grasping was performed with the hand supinated or pronated compared with the neutral position. This result is consistent with previous evidence in nonhuman primates showing that motor cortical cell activity changes according to the direction of a finger movement (Schwartz, 1992) and the position of the wrist joint (Thach, 1978). Indeed, in human subjects corticospinal excitability in hand muscles is modulated in a distinct manner by changing the orientation of the wrist and shoulder (Dominici et al., 2005; Gagné and Schneider, 2007). Evidence also shows that the neural drive to FDI changes according to the mechanical effectiveness of this muscle (Hudson et al., 2009). Thus, in our study grasping the object with minimal EMG activity in both planes might result in different challenges for the FDI muscle in terms of stability. With the hand in the supinated or pronated positions, the safety factor for the object slipping is quite small, because the "slip exit" between the fingers and the object is narrower than the center of the gripping area, whereas in the neutral position there is a larger possibility for slipping the object since the slip exit is larger. The reduced control needed during pronation/supination to prevent slipping may mean that 
the corticospinal contribution to the task is reduced. Another possibility is that the observed changes are related to modifications in muscle length and tension. This is supported by evidence showing that the corticospinal pathway may control motor actions by shifting spatial frames of reference during different upper-limb positions at similar levels of background EMG activity (Raptis et al., 2010). The changes in the interdependence of muscle forces between fingers during grip may also lead to the observed changes in corticospinal excitability. However, the similarities in the CV in the FDI and APB muscles suggest that it is less likely that small adjustments in index finger and thumb forces across tasks contributed to our results. Also, when MEPs were tested controlling for changes in posture and gravity, by asking subjects to perform unopposed index finger forces in the same plane as in the precision grip, no changes in MEP size were found making this possibility less likely.

\section{Neuronal mechanisms}

We found a decrease in intracortical inhibition as measured by the svEMG during grasping when the hand was oriented in pronation and supination compared with the neutral position. This result agrees with previous findings showing that activity in intracortical circuits is modulated during hand motor skills (Nordstrom and Butler, 2002). Thus, hand posture could directly modulate the excitability of intracortical inhibitory neurones, reducing inhibition and increasing direct control of cortical output to the FDI muscle. In a previous paper (Bunday et al., 2014), we argued that a smaller MEP during precision grip was due to suppression at spinal cord of indirect contributions to the MEP which increased the importance of direct corticospinal control. Reduced svEMG could thus reflect reduced inhibitory control of this output in parallel with its increased importance in precision grip.

It could also be argued that the activity in spinal cord circuits might contribute to the direction of grasping. Evidence has shown that the activity of some spinal interneurons shows a clear preferred direction during a force generation task (Shalit et al., 2012). However, we found no changes in the size of CMEPs across tasks. MEPs evoked by cervicomedullary stimulation activate the axons of pyramidal tract cells in the subcortical white matter and likely reflect changes occurring at subcortical levels (Taylor and Gandevia, 2004). The firing rate of motor units is determined by spinal mechanisms (Lüscher et al., 1979) and this is not affected by force direction at small levels of FDI muscle contraction (Enoka et al., 1989). We also found no changes in the amplitude and persistence of the F-waves in all muscles across tasks. The results of the F-wave measured during voluntary activity are difficult to interpret because an increase in motoneuron excitability by voluntary contraction increases the amplitude of the H-reflex and decrease the number of motoneurons able to produce an F-wave due to the increasing number of collisions between antidromic action potentials and H-reflex discharges in slowly conducting motor axons (Hultborn and Nielsen, 1995). Although, F-waves are produced by a limited number of motoneurons and commonly involve higher threshold motoneurons (Espiritu et al., 2003), whereas CMEPs recruit lower threshold motoneurons, the fact that CMEP size and F-waves remained similar across conditions, supports the view that it is less likely that the decrease in MEP size was related to changes occurring at spinal motoneurons. Because MEPs evoked by TMS and by cervicomedullary stimulation activates some of the same corticospinal axons (Taylor et al., 2002), it is likely that our results involve a cortical origin.
In conclusion, we previously showed (Bunday et al., 2014), using the FDI muscle, that the gain of motor cortical inputs to spinal motoneurons during precision grip was modulated via GABAb-ergic mechanisms within the spinal cord. The present results demonstrate that superimposed on this overall control is a cortical pattern of modulation in which the excitability of corticospinal output to FDI depends on the orientation of the hand relative to the body. In contrast, corticospinal excitability during unopposed finger flexion is not modulated by hand posture. The use of external reference frame parameters related to hand action might represent an avenue to shape excitability in cortical circuits during skilled human movement.

\section{References}

Bunday KL, Tazoe T, Rothwell JC, Perez MA (2014) Subcortical control of precision grip after human spinal cord injury. J Neurosci 34:7341-7350. CrossRef Medline

Butler JE, Larsen TS, Gandevia SC, Petersen NT (2007) The nature of corticospinal paths driving human motoneurones during voluntary contractions. J Physiol 584:651-659. CrossRef Medline

Buys EJ, Lemon RN, Mantel GW, Muir RB (1986) Selective facilitation of different hand muscles by single corticospinal neurones in the conscious monkey. J Physiol 381:529-549. CrossRef Medline

Chao EY, Opgrande JD, Axmear FE (1976) Three-dimensional force analysis of finger joints in selected isometric hand functions. J Biomech 9:387396. CrossRef Medline

Dominici F, Popa T, Ginanneschi F, Mazzocchio R, Rossi A (2005) Corticomotoneuronal output to intrinsic hand muscles is differentially influenced by static changes in shoulder positions. Exp Brain Res 164:500-504. CrossRef Medline

Enoka RM, Robinson GA, Kossev AR (1989) Task and fatigue effects on low-threshold motor units in human hand muscle. J Neurophysiol 62: 1344-1359. Medline

Espiritu MG, Lin CS, Burke D (2003) Motoneuron excitability and the F wave. Muscle Nerve 27:720-727. CrossRef Medline

Fetz EE, Perlmutter SI, Prut Y (2000) Functions of mammalian spinal interneurons during movement. Curr Opin Neurobiol 10:699-707. CrossRef Medline

Gagné M, Schneider C (2007) Dynamic changes in corticospinal control of precision grip during wrist movements. Brain Res 1164:32-43. CrossRef Medline

Georgopoulos AP, Kalaska JF, Caminiti R, Massey JT (1982) On the relations between the direction of two-dimensional arm movements and cell discharge in primate motor cortex. J Neurosci 2:1527-1537. Medline

Hudson AL, Taylor JL, Gandevia SC, Butler JE (2009) Coupling between mechanical and neural behaviour in the human first dorsal interosseous muscle. J Physiol 587:917-925. CrossRef Medline

Hultborn H, Nielsen JB (1995) H-reflexes and F-responses are not equally sensitive to changes in motoneuronal excitability. Muscle Nerve 18:14711474. CrossRef Medline

Kakei S, Hoffman DS, Strick PL (1999) Muscle and movement representations in the primary motor cortex. Science 285:2136-2139. CrossRef Medline

Lemon RN (2008) Descending pathways in motor control. Annu Rev Neurosci 31:195-218. CrossRef Medline

Lüscher HR, Ruenzel P, Henneman E (1979) How the size of motoneurones determines their susceptibility to discharge. Nature 282:859-861. CrossRef Medline

Mahan MY, Georgopoulos AP (2013) Motor directional tuning across brain areas: directional resonance and the role of inhibition for directional accuracy. Front Neural Circuits 7:92. CrossRef Medline

Maier MA, Hepp-Reymond MC (1995) EMG activation patterns during force production in precision grip: I. Contribution of 15 finger muscles to isometric force. Exp Brain Res 103:108-122. CrossRef Medline

Nordstrom MA, Butler SL (2002) Reduced intracortical inhibition and facilitation of corticospinal neurons in musicians. Exp Brain Res 144:336342. CrossRef Medline

Raptis H, Burtet L, Forget R, Feldman AG (2010) Control of wrist position and muscle relaxation by shifting spatial frames of reference for motoneuronal recruitment: possible involvement of corticospinal pathways. J Physiol 588:1551-1570. CrossRef Medline 
Rothwell JC, Hallett M, Berardelli A, Eisen A, Rossini P, Paulus W (1999) Magnetic stimulation: motor evoked potentials. The International Federation of Clinical Neurophysiology. Electroencephalogr Clin Neurophysiol Suppl 52:97-103. Medline

Rouiller EM, Yu XH, Moret V, Tempini A, Wiesendanger M, Liang F (1998) Dexterity in adult monkeys following early lesion of the motor cortical hand area: the role of cortex adjacent to the lesion. Eur J Neurosci 10:729740. CrossRef Medline

Sakai K, Ugawa Y, Terao Y, Hanajima R, Furubayashi T, Kanazawa I (1997) Preferential activation of different I waves by transcranial magnetic stimulation with a figure-of-eight-shaped coil. Exp Brain Res 113:24-32. CrossRef Medline

Schwartz AB (1992) Motor cortical activity during drawing movements: single-unit activity during sinusoid tracing. J Neurophysiol 68:528-541. Medline

Shalit U, Zinger N, Joshua M, Prut Y (2012) Descending systems translate transient cortical commands into a sustained muscle activation signal. Cereb Cortex 22:1904-1914. CrossRef Medline

Takei T, Seki K (2010) Spinal interneurons facilitate coactivation of hand muscles during a precision grip task in monkeys. J Neurosci 30:1704117050. CrossRef Medline

Takei T, Seki K (2013) Spinal premotor interneurons mediate dynamic and static motor commands for precision grip in monkeys. J Neurosci 33 : 8850 - 8860. CrossRef Medline

Taylor JL, Gandevia SC (2004) Noninvasive stimulation of the human corticospinal tract. J Appl Physiol 96:1496-1503. CrossRef Medline

Taylor JL, Petersen NT, Butler JE, Gandevia SC (2002) Interaction of transcranial magnetic stimulation and electrical transmastoid stimulation in human subjects. J Physiol 541:949-958. CrossRef Medline

Thach WT (1978) Correlation of neural discharge with pattern and force of muscular activity, joint position, and direction of intended next movement in motor cortex and cerebellum. J Neurophysiol 41:654-676. Medline 